\title{
EULAR recommendations for the management of familial Mediterranean fever
}

\author{
Seza Ozen, ${ }^{1}$ Erkan Demirkaya, ${ }^{2}$ Burak Erer, ${ }^{3}$ Avi Livneh, ${ }^{4}$ Eldad Ben-Chetrit, ${ }^{5}$ \\ Gabriella Giancane, ${ }^{6}$ Huri Ozdogan, ${ }^{7}$ Illana Abu, ${ }^{8}$ Marco Gattorno, ${ }^{9}$ \\ Philip N Hawkins, ${ }^{10}$ Sezin Yuce, ${ }^{11}$ Tilmann Kallinich, ${ }^{12}$ Yelda Bilginer, ${ }^{13}$ \\ Daniel Kastner, ${ }^{14}$ Loreto Carmona ${ }^{15}$
}

\begin{abstract}
Handling editor Tore K Kvien
- Additional material is published online only. To view please visit the journal online (http://dx.doi.org/10.1136/ annrheumdis-2015-208690)

For numbered affiliations see end of article.

\section{Correspondence to} Professor Seza Ozen, Department of Pediatric Rheumatology, Hacettepe University Faculty of Medicine, Ankara 06100, Turkey; sezaozen@hacettepe.edu.tr
\end{abstract}

Received 25 November 2015 Revised 1 January 2016 Accepted 3 January 2016 Published Online First 22 January 2016
CrossMark

To cite: Ozen $S$,

Demirkaya E, Erer B, et al. Ann Rheum Dis

2016;75:644-651

\section{ABSTRACT}

Familial Mediterranean fever (FMF) is the most common monogenic autoinflammatory disease, but many rheumatologists are not well acquainted with its management. The objective of this report is to produce evidence-based recommendations to guide rheumatologists and other health professionals in the treatment and follow-up of patients with FMF. A multidisciplinary panel, including rheumatologists, internists, paediatricians, a nurse, a methodologist and a patient representative, was assembled. Panellists came from the Eastern Mediterranean area, Europe and North America. A preliminary systematic literature search on the pharmacological treatment of FMF was performed following which the expert group convened to define aims, scope and users of the guidelines and established the need for additional reviews on controversial topics. In a second meeting, recommendations were discussed and refined in light of available evidence. Finally, agreement with the recommendations was obtained from a larger group of experts through a Delphi survey. The level of evidence (LoE) and grade of recommendation (GR) were then incorporated. The final document comprises 18 recommendations, each presented with its degree of agreement (0-10), LoE, GR and rationale. The degree of agreement was greater than $7 / 10$ in all instances. The more controversial statements were those related to follow-up and dose change, for which supporting evidence is limited. A set of widely accepted recommendations for the treatment and monitoring of FMF is presented, supported by the best available evidence and expert opinion. It is believed that these recommendations will be useful in guiding physicians in the care of patients with FMF.

\section{INTRODUCTION}

Autoinflammatory diseases (AIDs) are rare clinical conditions, of which familial Mediterranean fever (FMF) is the most common. Unfortunately, medical curricula and many rheumatological centres do not cover these syndromes adequately, resulting in lack of knowledge in identifying the optimal management of these patients. In general, FMF can be well controlled with appropriate use of medications and monitoring. However, different views on management exist depending on the experience of the caring physician, the geographic area and available health resources, which can impact negatively on clinical outcome. Disparities in the management of diseases are not acceptable if unrelated to the severity of the underlying phenotype, especially when efficacious and cost-effective treatment exists. Attempts to resolve practical questions in the daily management of patients with FMF have been published, ${ }^{1}{ }^{2}$ but these guidelines have addressed only limited aspects of management, most particularly colchicine therapy, and have overlooked other important facets of management.

An international collaboration of experienced experts from numerous countries advocated these recommendations. The objective was to guide physicians who are not experts in the disease in a wide range of management aspects, by exploring and then establishing evidence-based recommendations from a multidisciplinary perspective.

The scope of these recommendations includes FMF itself, its complications and comorbidities that may affect its management. The users are expected to be physicians-mainly rheumatologists and paediatricians - and other healthcare professionals who care for patients with FMF. It is hoped that these recommendations will also be used by policymakers and health authorities with financial responsibility for the care of these patients.

As per the overarching principles, the recommendations are evidence based to the greatest possible extent. However, in areas for which limited information exists, a pragmatic consensus of expert opinion has also been employed.

\section{METHODS}

For these recommendations, we used the following methodologies: discussion group, systematic reviews and Delphi technique. These recommendations were developed according to the European League Against Rheumatism (EULAR) standardised operating procedures (SOPs), including the categorisation of evidence. ${ }^{3}$

The discussion group was formed by a multinational multidisciplinary panel, including rheumatologists, internists, paediatricians, a nurse, a methodologist and a patient representative. There were 10 experts and a patient representative on the panel. The panel was moderated by a methodologist (LC) and determined the users, aims and scope of the recommendations, along with the structure of the document. Systematic reviews were performed prior to the panel discussions. These analysed available evidence on efficacy and safety of treatments in FMF and reviewed the incidence of complications, including amyloidosis, renal failure and infertility. In addition, during the discussion, the panel decided to review the contribution of 
serum amyloid A (SAA) protein and C reactive protein (CRP) to management and predicting complications. The systematic reviews were performed by three fellows (ED, BE, GG) under the supervision of the methodologist. The methods and full review of two subject areas (efficacy and acute phase reactants (APR)) have been published. ${ }^{45}$

The experts formulated practical recommendations during the first meeting and clarified the rationale for the statements. All recommendations and rationales were structured as a matrix enabling on-line comments from all collaborators. The document was discussed in a second meeting, and votes were taken on the recommendations. When there was high discordance (a SD greater than 2 or an IQR greater than 5), the recommendation was discussed and reformulated, and when there was agreement against the recommendation, it was dropped. Finally, the document was refined and reformatted to improve readability and understanding. The methodologist added the level of evidence (LoE) and grade of recommendation to each statement, based on the Oxford Centre for Evidence Based Medicine guidelines. ${ }^{6}$ In parallel, the recommendations were converted into items of a Delphi survey and submitted to 67 experts, included the panel (listed in online supplementary appendix), to determine the level of agreement. Agreement was graded from 0 - 'no agreement' to 10 -'maximum agreement'.

\section{RESULTS}

The recommendations are presented in text with the rationale plus in table 1, with the LoE and agreement by a large group of experts.

1. Ideally, FMF should be diagnosed and initially treated by a physician with experience in FMF.
FMF can be treated by different experienced specialists, namely clinical geneticists, paediatric and adult rheumatologists, internists, nephrologists and gastroenterologists. A specialist with experience in FMF is a physician usually working in a referral centre who is involved in the clinical care of patients with FMF and is capable of dealing with difficult cases and other AIDs that are potential differential diagnoses. Following diagnosis and initiation of therapy, patients can also be followed by their general practitioner or paediatrician in conjunction with the referral centre. It is recommended that, if possible, patients are reviewed by a physician with experience of FMF at least once per year in the long term.

2. The ultimate goal of treatment in FMF is to obtain complete control of unprovoked attacks and minimise subclinical inflammation in between attacks.

There are two main goals in the treatment of FMF. The first is to prevent the clinical attacks and the second is to suppress chronic subclinical inflammation and elevation of APR, in particular SAA protein, and its consequences, including amyloid A (AA) (secondary) amyloidosis and other long-term complications. Improved quality of life by reducing the attacks is an achievable target in most patients. However, complete cessation of attacks may not be possible in patients with more severe forms of FMF, notably including many of those who are homozygous for M694V. Very importantly, the development of AA amyloidosis can be prevented when treatment substantially maintains normal SAA protein concentration between attacks. This is an especially important objective in patients with a family history of AA amyloidosis. ${ }^{7-11}$ Biological treatment such as anti-interleukin 1 (IL-1) therapy should be considered if the inflammation cannot be controlled with adequate colchicine (see below).

Table 1 EULAR recommendations for the management of FMF with the level of agreement, of evidence and grade of recommendation (GR)

\begin{tabular}{|c|c|c|c|}
\hline Recommendation & A & LoE & GR \\
\hline 01. Ideally, FMF should be diagnosed and initially treated by a physician with experience in FMF & 7.6 & 5 & D \\
\hline 02. The ultimate goal of treatment in FMF is to reach complete control of unprovoked attacks and minimising subclinical inflammation in between attacks & 9.3 & 4 & C \\
\hline 04. Dosing can be in single or divided doses, depending on tolerance and compliance & 9.4 & 5 & D \\
\hline 05. The persistence of attacks or of subclinical inflammation represents an indication to increase the colchicine dose & 9.7 & 3 & C \\
\hline $\begin{array}{l}\text { 06. Compliant patients not responding to the maximum tolerated dose of colchicine can be considered non-respondent or resistant; alternative biological } \\
\text { treatments are indicated in these patients }\end{array}$ & 9.8 & $2 \mathrm{~b}$ & B \\
\hline 08. Periods of physical or emotional stress can trigger FMF attacks, and it may be appropriate to increase the dose of colchicine temporarily & 7.6 & 5 & D \\
\hline 09. Response, toxicity and compliance should be monitored every 6 months & 8.6 & 5 & D \\
\hline $\begin{array}{l}\text { 10. Liver enzymes should be monitored regularly in patients with FMF treated with colchicine; if liver enzymes are elevated greater than twofold the upper } \\
\text { limit of normal, colchicine should be reduced and the cause further investigated }\end{array}$ & 8.4 & 5 & D \\
\hline $\begin{array}{l}\text { 11. In patients with decreased renal function, the risk of toxicity is very high, and therefore signs of colchicine toxicity, as well as CPK, should be carefully } \\
\text { monitored and colchicine dose reduced accordingly }\end{array}$ & 9.3 & 4 & C \\
\hline $\begin{array}{l}\text { 15. In general, men do not need to stop colchicine prior to conception; in the rare case of azoospermia or oligospermia proven to be related to colchicine, } \\
\text { temporary dose reduction or discontinuation may be needed }\end{array}$ & 8.2 & 3 & C \\
\hline 16. Chronic arthritis in a patient with FMF might need additional medications, such as DMARDs, intra-articular steroid injections or biologics & 9.5 & $2 b$ & C \\
\hline $\begin{array}{l}\text { 17. In protracted febrile myalgia, glucocorticoids lead to the resolution of symptoms; NSAID and IL-1-blockade might also be a treatment option; NSAIDs } \\
\text { are suggested for the treatment of exertional leg pain }\end{array}$ & 9.3 & $2 b$ & $\mathrm{C}$ \\
\hline 18. If a patient is stable with no attacks for more than 5 years and no elevated APR, dose redu & 8.0 & 5 & D \\
\hline
\end{tabular}

A, agreement (/10); APR, acute phase reactants; CPK, creatinine phosphokinase; DMARDs, disease modifying antirheumatic drugs; EULAR, European League Against Rheumatism; FMF, familial Mediterranean fever; IL-1, interleukin 1; LoE, level of evidence; NSAID, non steroidal anti inflammatory drugs. 
3. Treatment with colchicine should be started as soon as a clinical diagnosis is made.

Colchicine is very efficacious in preventing FMF attacks and associated amyloidosis. ${ }^{4}{ }^{12-14} \mathrm{~A}$ starting dose of $\leq 0.5 \mathrm{mg} / \mathrm{day}$ $(\leq 0.6 \mathrm{mg} /$ day in case tablets contain $0.6 \mathrm{mg})$ for children $<5$ years of age, $0.5-1.0 \mathrm{mg} /$ day $(1.2 \mathrm{mg} /$ day in case tablets contain $0.6 \mathrm{mg}$ ) for children $5-10$ years of age, $1.0-1.5 \mathrm{mg} / \mathrm{day}$ $(1.8 \mathrm{mg} /$ day in case tablets contain $0.6 \mathrm{mg})$ in children $>10$ years of age and in adults is recommended. ${ }^{2}{ }^{13-15} \mathrm{In}$ patients with pre-existing complications (eg, amyloidosis) or greater disease activity, higher doses may be initiated.

The remarkable efficacy of colchicine in FMF underscores its role as a diagnostic tool in suspected cases, that is, supporting its introduction before the diagnosis is certain. ${ }^{12}$ On the other hand, there are instances in which a delay in commencing colchicine treatment for a short period of observation may be informative, potentially enabling an attack to be observed, but most experts in the panel were not comfortable with this practice. After colchicine has been commenced, patients should be followed closely for 3-6 months to observe its therapeutic effect on attack frequency and severity. Most experts prefer to start with low and increase the dose according to the patient's response and tolerance ${ }^{16}$ (see recommendation 5 on dose increment).

A genetic diagnosis of FMF in the absence of clinical manifestations or subclinical inflammation is not necessarily an indication to start treatment, but such patients must remain under surveillance since they may develop clinically significant disease in future, even without symptoms. In countries where secondary amyloidosis is frequent, the physician may consider treatment, especially if there are similar cases in the family. Homozygosity for the M694V genotype among symptomatic patients, which is more frequently associated with the development of amyloidosis $^{17-25}$ and a requirement for higher doses of colchicine, ${ }^{20} 2325$ is considered by some experts to be an indication for commencing a higher dose of colchicine than the general recommendation above.

4. Dosing can be in single or divided doses, depending on tolerance and compliance.

Colchicine has an excellent long-term safety profile, but is commonly associated with gastrointestinal side effects. ${ }^{26}$ Therapeutic oral doses of colchicine may cause cramping, abdominal pain, hyperperistalsis, diarrhoea and vomiting, which may be transient or persistent. A single daily dose regimen may increase compliance, but the dose can be divided to diminish side effects. An association of lactose intolerance and diarrhoea has been reported. ${ }^{27}$ Dietary modification (ie, temporary reduction of dairy products), split doses, dose reduction and antidiarrhoeal and spasmolytic agents may be recommended. Once symptoms resolve, the regular prophylactic dosage needs to be reintroduced in a gradual stepwise fashion. To overcome these problems, treatment with colchicine can be started at the usually subtherapeutic dose of $0.5 \mathrm{mg} /$ day and increased gradually by $0.5 \mathrm{mg}$ in divided daily doses. ${ }^{28}$ In more difficult cases, oral desensitisation similar to that used in cases of allergic reactions may be attempted. ${ }^{29}{ }^{30}$ Colchicine had been used parenterally by weekly intravenous injection in critically ill patients, but this is associated with substantially increased risk of toxicity. ${ }^{31} 32$

5. The persistence of attacks or subclinical inflammation represents an indication to increase colchicine dose.

If inflammation persists despite adherence to the advised initial dose of colchicine, as defined by continuing attacks or elevated APR between attacks, colchicine dose may be increased by $0.5 \mathrm{mg} / \mathrm{day}$ (or $0.6 \mathrm{mg} / \mathrm{day}$ depending on the available drug formulation) with careful monitoring of side effects. Colchicine may be increased up to a daily dose of $2 \mathrm{mg}$ in children and $3 \mathrm{mg}$ in adults, or the maximum tolerated dose if this cannot be appropriate. Monitoring CRP, SAA protein or both at least every 3 months is required during dose escalation in patients with active disease to determine the necessary colchicine dose. Disease severity and patients' tolerance of FMF attacks should also be taken into account in establishing a tailored colchicine dose.

6. Compliant patients not responding to the maximum tolerated dose of colchicine can be considered non-responders or resistant; alternative biological treatments are indicated in these patients.

Patients who continue to have one or more attacks each month despite receiving the maximally tolerated dose for at least 6 months may be considered to be a non-responder or resistant to colchicines, although compliance to treatment must be affirmed. In addition, there are patients who do not tolerate even infrequent attacks or have evidence of significant persistent subclinical inflammation leaving them at risk of developing amyloidosis. Evidence for therapeutic options for patients resistant or intolerant to colchicine is limited(reference in press), but case reports and case series have suggested that IL- 1 blockade is a promising second-line therapy. ${ }^{33} 34$ This is particularly important in patients with a family history of AA amyloidosis. A recent small randomised controlled trial $(n=14)$ of the IL-1 blocker rilonacept in colchicine-resistant patients with FMF reported a significant reduction in the number of attacks. ${ }^{35}$ Phase III trials with canakinumab and anakinra are currently being conducted. ${ }^{36}{ }^{37}$ Tumour necrosis factor (TNF) inhibitors have also been used in colchicine-resistant patients, especially with articular involvement, with good responses reported in observational studies. $^{38} 39$

Some experts use biological therapy only temporarily, although this approach has not been studied formally. It is recommended that colchicine should be coadministered with alternative biological therapies given that it may reduce the risk of amyloidosis despite persistence of attacks. ${ }^{14}$

7. FMF treatment needs to be intensified in AA amyloidosis using the maximal tolerated dose of colchicine and supplemented with biologics as required.

AA amyloidosis is the most serious complication of uncontrolled FMF, resulting in renal failure and early death. ${ }^{40}$ The development of amyloidosis can be prevented in most patients by suppressing chronic inflammatory activity with the measures already discussed. Furthermore, complete suppression of inflammatory activity has potential to prevent progression or reverse established amyloidosis. ${ }^{11}$

The median latency between onset of inflammatory disease and diagnosis of AA amyloidosis is approximately 17 years, although this varies enormously. ${ }^{41}$ The predominant clinical manifestation of AA amyloidosis is renal dysfunction, with the majority of patients presenting with proteinuric kidney disease. $^{42}$ Renal biopsy is required to confirm the diagnosis of amyloidosis in patients with FMF with proteinuria. In a report, $1.7 \%$ of patients with FMF on colchicine developed proteinuria, while it occurred in $48 \%$ of untreated patients. ${ }^{11}$ In 5/86 (5.81\%) patients, overt proteinuria has been reported to disappear. ${ }^{11}$ Progression to dialysis eventually occurs in $50 \%$ of patients with proteinuria. The spleen is affected in almost all cases and the adrenal glands in at least a third, although clinically relevant hypoadrenalism is uncommon; the liver and gut are also frequent sites of AA amyloid deposition, but the heart is rarely involved. ${ }^{42}$ 
Treatment for amyloidosis comprises measures to support failing organ function, including blood pressure control and dialysis for patients with renal disease. Amyloid deposits are naturally turned over only very slowly, but a response to colchicine or biological treatment in FMF that reduces the supply of SAA protein, the AA amyloid fibril precursor protein, may facilitate net regression of the amyloid deposits. This may lead to preservation or slow recovery of amyloidotic organ function. Colchicine must be given in doses that are sufficient to produce sustained control of the inflammatory disease in order to prevent ongoing abnormal production of SAA protein. Therapeutic success is ideally monitored by frequent estimation of SAA protein with the target being maintenance below $10 \mathrm{mg} / \mathrm{L}$ and by assessment of proteinuria and glomerular filtration. The measurement of CRP is an imperfect surrogate for SAA protein should the latter assay be unavailable. Amyloidotic kidneys are unusually sensitive to additional injury by hypoperfusion, hypertension, nephrotoxic drugs and surgery, all of which should be avoided as far as practicable.

The majority of patients with FMF and amyloidosis will eventually require renal replacement therapy and survival on dialysis is now comparable with that of non-diabetic-associated endstage renal failure. ${ }^{43}$ Recent experience of renal transplantation in selected patients has been encouraging with long-term graft and patient survival matching that of the age-matched general transplant population. ${ }^{4-46}$ These encouraging outcomes have led to increasing use of living donor renal transplants.

8. Periods of physical or emotional stress can trigger FMF attacks, and it may be worth temporarily increasing the dose of colchicine.

Efforts in individual patients to identify factors that trigger attacks may help to prevent or reduce them. Recognised trigger factors include physical or emotional stress, menstruation, physical trauma, exposure to cold, infections, inflammation, high fat intake, starvation, sleeplessness and tiredness. ${ }^{47}$ These triggers may differ depending on the type of attack. ${ }^{48} 49$ Some authors recommend increasing the dose of colchicine to pre-empt trigger events. 5051

9. Response, toxicity and compliance should be monitored every 6 months.

There is neither agreement on the definition of adequate response nor consensus on the number of attacks per year that may be considered 'acceptable'; this tolerability level should be judged in the context of the quality of life of the patient. Experts recommend reviewing patients at 6-monthly intervals to evaluate the frequency and character of the attacks, as well as to monitor the APR response in between attacks. During the first year, patients may need to be monitored more frequently to assess tolerability of treatment, as well as potential side effects and compliance. More frequent evaluations may be needed to adjust treatment, especially in children in whom frequent blood sampling may not be practical. Adverse events other than diarrhoea, abdominal cramps, possible sperm reduction and mild abnormalities of liver enzymes-all discussed elsewhere in this document-are extremely rare but include alopecia, neutropenia and peripheral neuropathy. Adverse effects of colchicine might be ameliorated by dose reduction, but the likelihood and risks of precipitating greater FMF activity must be considered.

Patients may also need more frequent assessments if APR are repeatedly elevated, the disease is unstable, at any time when the dose is being adjusted or suspected toxicity develops. By contrast, the intervals can be increased to yearly when patients are stable.

Response could be monitored with the Auto-Inflammatory Diseases Activity Index (AIDAI), a diary in which patient- reported features such as temperature and abdominal pain are recorded and a disease activity score is calculated. ${ }^{52}$ Juvenile Autoinflammatory Disease Multidimensional Assessment Report (JAIMAR) is a qualitative assessment for AIDs that may also be used. $^{53}$

Laboratory tests are recommended to monitor liver enzymes, complete cell blood count, kidney function, creatinine phosphokinase (CPK) and to identify proteinuria. The preferred APR are SAA protein and CRP. ${ }^{8} 54$

Compliance with colchicine taken on a regular daily basis is the cornerstone of management of FMF, with potential to reduce the frequency and severity of clinical attacks and prevent the development of AA amyloidosis, renal failure and premature death in most patients. ${ }^{55}$ There is, however, a surprisingly high rate of poor compliance with colchicine therapy among affected patients. Reasons offered by patients for failing to take colchicine on the required daily basis include non-specific concerns about potentially lifelong use of the drug, concerns about adverse effects such as bloating and diarrhoea, concerns about fertility and sexual function and concerns about harm to unborn children. Among adolescent patients, deviation from the prescribed dose may be a feature of deliberately manipulative behaviour but more commonly is associated with inconvenience, fear of side effects, embarrassment and laziness.

Unfortunately, there is no accessible assay to determine the concentration of colchicine in the blood, and lack of compliance should be considered in all patients with FMF in whom colchicine appears to be ineffective in preventing attacks or the development of amyloidosis.

10. Liver enzymes should be monitored regularly in patients with FMF treated with colchicine; if liver enzymes are elevated greater than twofold the upper limit of normal, colchicine should be reduced and the cause further investigated.

Liver enzymes can become elevated in patients with FMF receiving colchicine, for reasons that are not always clear. In a minority of cases, discontinuation of colchicine will lead to normalisation of the enzyme values, but in most there proves to be no clear association with the drug. Tweezer-Zaks et al, reported that cryptogenic cirrhosis is more common among patients with FMF compared with its prevalence in the general population. ${ }^{56}$ Rimar et $a l^{57}$ reported that non-alcoholic cirrhosis is more common in FMF, perhaps as a result of uncontrolled inflammation. Thus, significantly elevated liver enzymes should be investigated for causes other than an adverse effect of colchicine treatment.

11. In patients with decreased renal function, the risk of colchicine toxicity is very high and therefore evidence of toxicity should routinely be sought and the colchicine dose reduced accordingly.

Patients with FMF may develop impaired renal function due to amyloidosis or other causes. ${ }^{7}$ There is no specific cut-off for reduced glomerular filtration rate to guide colchicine dose reduction, but it has been shown that patients with FMF on colchicine with elevated creatinine are prone to develop muscle pain with myopathy. ${ }^{58}$ In such cases, elevation of CPK can help guide colchicine dose reduction.

Colchicine is not removed significantly during haemodialysis. While high-flux polysulfone filters can clear colchicine from the blood more effectively than conventional dialysers, their efficiency is insufficient to treat colchicine overdose or toxicity. ${ }^{59}$

12. Colchicine toxicity is a serious complication that should be given adequate consideration and be prevented.

Colchicine is an alkaloid with a narrow therapeutic range. ${ }^{26}{ }^{60}$ High concentrations may cause serious toxicity that 
can be life threatening. Since there are no effective means to remove colchicine from the tissues and the blood, efforts must be made to avoid overdose and toxicity.

Conditions that may lead to colchicine toxicity are as follows:

- Exceeding the recommended dose: the maximum recommended oral doses for treatment of FMF are $3 \mathrm{mg}$ daily in adults and 2 mg daily in children. ${ }^{1}$

- Liver or renal failure: colchicine is partially metabolised in the liver and its metabolites are excreted mainly through the biliary tract and the kidneys. After oral ingestion of pharmacological doses, the mean elimination half-life is $9-16 \mathrm{~h}$, but this may be up to sevenfold longer in patients with liver cirrhosis. $^{61}$

- Concomitant administration of other drugs (macrolides, ketoconazole, ritonavir, verapamil, ciclosporin, statins or other drugs metabolised by cytochrome 3A4): potential drug-drug interactions and colchicine may increase its blood levels by $200-300 \% .^{62}$ The use of macrolides in children and ciclosporin in transplanted patients warrants special caution and the primary physician should be informed about the interactions.

Colchicine overdose may cause abdominal cramping, vomiting and diarrhoea. ${ }^{63}$ In the first stage, colchicine toxicity may manifest as gastrointestinal symptoms with a cholera-like syndrome associated with dehydration, shock, acute renal failure, hepatocellular failure and even seizures. ${ }^{64}$ The second stage develops $24-72 \mathrm{~h}$ following ingestion of the drug and is dominated by multiorgan failure. This may include bone marrow failure, renal insufficiency, adult respiratory distress syndrome, arrhythmias, disseminated intravascular coagulation, neuromuscular disturbances, coma and death. If the patient survives this stage, which may last several weeks, he or she may enter the third stage, which is characterised by recovery of bone marrow and rebound leukocytosis, resolution of organ failure and alopecia.

Clinical management of colchicine toxicity is essentially supportive. In a single case, treatment with $\mathrm{F}(\mathrm{ab})$ fragments of anticolchicine antibodies was used successfully, ${ }^{65}$ but since this antidote is not generally available, colchicine toxicity may be fatal.

13. When suspecting an attack, always consider other possible causes. During the attacks, continue the usual dose of colchicine and use non steroidal anti inflammatory drugs (NSAIDs).

On should verify whether the symptoms of the patient are indeed due to FMF and should ask the patient whether it resembles previous attacks. In unclear situations, monitor APR over several hours and consider relevant imaging, for example, radiography for chest pain. Patients with FMF are as susceptible to appendicitis as anyone else!

Symptoms during attacks may be alleviated by NSAIDs (naproxen, diclofenac, indomethacin, etc). ${ }^{66}$ Glucocorticoids may decrease the duration of attacks, but may also increase their frequency. ${ }^{67}$ There is no clear evidence on the efficacy of shortterm administration of IL-1 blockers during attacks. ${ }^{68}$ Many patients report that a temporary increase in colchicine dose during a prodrome may shorten or prevent attacks, although this has not been proven.

14. Colchicine should not be discontinued during conception, pregnancy or lactation; current evidence does not justify amniocentesis.

Patients need to be informed about the safety of colchicine treatment during conception, pregnancy and breast feeding. According to some manufacturer brochures, treatment with colchicine during pregnancy and nursing is contraindicated.
However, a systematic review did not identify a higher rate of neither abortion nor malformation in women with FMF receiving colchicine compared with healthy subjects (reference in press). On the other hand, the rate of abortion and miscarriages in women with FMF who are not receiving colchicine treatment is greater than expected. ${ }^{50}$ Discontinuation of colchicine may lead to exacerbation of FMF attacks and in long term to the development of amyloidosis. In pregnant women, an acute FMF attack with peritonitis may lead to premature contractions and early delivery or abortion. In a study in which colchicine was measured in the milk and the blood of nursing patients with FMF, it was shown that the neonates are exposed to only very small quantities of the drug, which cannot adversely affect them. ${ }^{69}$ Monitoring frequency and colchicine dose should be adjusted during pregnancy depending on FMF activity.

15. In general, men need not stop colchicine prior to conception; in the rare case of azoospermia or oligospermia proven to be related to colchicine, temporary dose reduction or discontinuation may be required.

Colchicine is a drug that may affect microtubules in various cells. In high concentrations, it may inhibit mitosis within the process of cell division. ${ }^{70}$ Some animal studies and case reports support the association with azoospermia, but only in very high doses. $^{71} 72$ Reassuringly, a cytogenetic evaluation in patients with FMF receiving long-term colchicine showed no differences compared with controls. ${ }^{73}$ Bremner and Paulsen have failed to show any effect on spermatogenesis in six healthy volunteers who received commonly used doses of colchicine for 4-6 months. ${ }^{74}$ Very probably, the frequency of oligospermia or azoospermia with colchicine depends on the underlying disease, and in FMF it may also be due to amyloidosis. ${ }^{75}$ Therefore, caution is required in evaluating the cause of azoospermia in patients with FMF and amyloidosis of the testes should be considered. When azoospermia is caused by colchicine, it can be discontinued and substituted by anti-IL- 1 treatment for about 3 months allowing recovery of the testes. Colchicine should be resumed after successful conception. ${ }^{76}$

16. Chronic arthritis in a patient with FMF might need additional medications, such as disease modifying antirheumatic drugs (DMARDs), intra-articular steroid injections or biologics.

Approximately 5\% of patients with FMF (160/3000) develop chronic joint involvement, the majority resembling spondyloarthritis with sacroiliitis and peripheral monoarthritis or oligoarthritis and rarely resembling juvenile idiopathic arthritis. Colchicine is not always effective in the treatment of chronic arthritis of FMF and such cases need to be treated with DMARDs and biological agents. $^{77} 78$

17. In protracted febrile myalgia, glucocorticoids lead to the resolution of symptoms; NSAID and IL-1 blockade might also be a treatment option. NSAIDs are suggested for the treatment of exertional leg pain.

Protracted febrile myalgia is defined as severe disabling myalgia of at least 5 days duration in a patient with FMF associated with fever, elevated levels of inflammatory markers and the presence of at least one M694V mutation. ${ }^{79} 80$ There is a striking contrast between the extreme severity of pain and tenderness in patients with the protracted febrile myalgia and the findings of normal CPK and subtle non-specific electromyography (EMG). ${ }^{81}$ Steroid treatment leads to prompt improvement. $^{79} 8182$ NSAID might also be beneficial. ${ }^{79}$ Two cases successfully treated by IL-1 blockade have been reported. ${ }^{83}$

18. If a patient is stable with no attacks for more than 5 years and no elevated APR, dose reduction could be considered after expert consultation and with continued monitoring. 
Colchicine-free remission in patients with FMF has been observed, ${ }^{84}$ usually among patients who hitherto had mild disease and have mutations associated with mild disease or with low penetrance-namely the absence of homozygosity to the mutations M694V, M680I, M694I and V726A-E148Q complex allele. A trial to reduce colchicine dose appears reasonable among such patients, particularly those who have been stable with no attacks for several years and have not had elevated APR. Dose reduction could be considered after expert consultation, and only if appropriate measures continue to be taken to identify subclinical inflammation and hence prevent the development of 'silent' amyloidosis. Dose decrement must be performed gradually by no more than $0.5 \mathrm{mg}$ on each occasion. The suggested interval for colchicine dose reduction is 6 months. Patients must remain under clinical observation and perform periodic laboratory evaluation of SAA protein or CRP and urinary protein. Blood tests should be performed 3 months after dose reduction. Colchicine dose reduction should be avoided in patients who may be unwilling to comply with these strict constraints. It must be stressed that dose reduction is appropriate only in a small minority of patients and considered extremely rare. Such a trial must be conducted by physicians with expertise in FMF.

\section{DISCUSSION}

These recommendations were developed by a formal and systematic methodology and by a multidisciplinary panel from many countries. The agreement is high and all controversies are discussed in detail. The overarching principles were evidence based and practical statements that covered as many aspects as possible of management after diagnosis. Although the guidelines have not been reviewed by external experts, it has many features that underscore its validity. The development group includes individuals from all the relevant professional groups and has also sought the views and preferences of the patient. Both the health benefits and the side effects and risks have been considered in formulating the recommendations. Furthermore, the Delphi group has been very widely chosen to collect the opinion of many experts around the world and in order to tackle ambiguity of the statements. Moreover, we can ensure editorial independence of the board, as very few pharmacological options are available in FMF and the main drug is cheap and has been available for many years; the exceptions have been discussed in a transparent form.

FMF is a chronic, lifelong inflammatory disease. It is increasingly recognised around the world due to increased awareness of AIDs and due to the migration of populations with a high carrier rate during the past century. A remarkable feature of FMF is the availability of a highly effective long-term oral drug therapy, colchicine, which was recognised and introduced in 1972. Colchicine is safe, can be taken as a single daily dose, is of relatively low cost and is widely available. The aim of colchicine therapy is twofold in FMF: decreasing the frequency and severity of clinical attacks and preventing the risk of developing AA amyloidosis. Unfortunately, there is no internationally agreed consensus on the definition of response or unresponsiveness, a clearly desirable aim for the FMF community.

AA amyloidosis, formerly known as secondary amyloidosis, is a complication of chronic inflammation and was a frequent complication of FMF in the pre-colchicine era, causing death from early adulthood onwards. Availability of colchicine has reduced the risk of developing amyloidosis from $60 \%$ to less than $13 \%$ in Turkey, but it remains a major cause of mortality in FMF even recently.
SHARE, which is a mainly European paediatric initiative, has suggested recommendations for the diagnosis and genetic understanding of the disease. ${ }^{85}$ In addition, in 2012, a group of clinicians and geneticists had developed guidelines for the analysis and reporting of the genetic results in the main AIDs, including FMF. $^{86}$

Measures that can improve compliance include provision of information specific to the large body of experience using colchicine in FMF during the past four decades. While gastrointestinal side effects are common, it can be stressed that these symptoms are harmless. There are a number of drug interactions with colchicine such as macrolide antibiotics and statins; thus, the primary physician should be informed that the patient is on colchicine treatment. The benefits of regular colchicine with respect to prevention of amyloidosis should be stressed firmly and repeatedly. This can be reinforced by performing urinalysis at all clinic visits to exclude proteinuria, and measurements of the AA amyloid precursor protein SAA, high values of which can be shared with the patient to further emphasise the need for compliance.

Patients with FMF may require additional treatment for the specific features highlighted in recommendations 16 and 17, such as protracted febrile myalgia and arthritis. Furthermore, in the rare patients who fail to respond to colchicine or who cannot tolerate it, biological drugs, especially anti-IL-1 treatment, should be considered. However, colchicine should be continued in patients with FMF who receive such treatments.

The studies that support these recommendations, as was evident during the systematic reviews, have many limitations that in part are related to the rare nature of FMF. The study designs are complicated due to the heterogeneity of the clinical phenotype, the sample sizes are small due to the rarity of the disease and the absence of uniform outcome measures complicate the design of clinical trials even more. Recommendations relating to research in the area of FMF need to (1) take into account the episodic nature of the disease in observational studies and use time-varying covariates, serial studies and adjustment for confounding factors; (2) explain the need to include healthy controls in most studies-this will depend on the research question and these questions are seldom related to the difference versus the general population, but to the natural course of disease; (3) have clearer research questions in general and (4) join efforts to increase sample size.

Although the level of agreement is high, we still detected controversy in many issues. This is mainly due to the low LoE and the heterogeneous and rare nature of the disease. As a result, many recommendations were not under category $\mathrm{A}$, and yet the experts were prompt to provide advice on critical matters with practical implications. In this sense, the experts should be commended for sharing the actual practice of many years.

Implementations is a critical issue of guidelines as reflected in the recent update of EULAR SOP (3). Indications of how to increase the implementations of these recommendations are (1) to include them in the EULAR textbook of rheumatology, (2) to present them in Congresses of the various specialties involved and (3) to facilitate the table with the recommendations to patient organisations.

These recommendations are very timely, as several new therapeutic alternatives are currently being studied for patients unresponsive to colchicine. ${ }^{34}$ Thus, it is hoped that they will be used by a large audience as discussed above. Given the ongoing translational and clinical studies, we anticipate the need for an update in the period of less than 5 years. 


\section{Author affiliations}

${ }^{1}$ Department of Pediatric Rheumatology, Hacettepe University, Ankara, Turkey

${ }^{2}$ Division of Pediatric Rheumatology, Gülhane Military Medical Academy, FMF Arthritis Vasculitis \& Orphan Disease Research Center (FAVOR), Ankara, Turkey ${ }^{3}$ Division of Rheumatology, Department of Internal Medicine, Istanbul University, Istanbul Faculty of Medicine, Istanbul, Turkey

${ }^{4}$ Heller Institute of Medical Research and Medicine F, Sheba Medical Center, TelHashomer and Sackler Faculty of Medicine, Tel-Aviv University, Tel Aviv, Israel

${ }^{5}$ Rheumatology Unit, Hadassah-Hebrew University Medical Center, Jerusalem, Israel

${ }^{6}$ Pediatria II-Reumatologia, Istituto G. Gaslini, Genova, Italy

${ }^{7}$ Rheumatology Unit, Cerrahpaşa Medical Faculty, İstanbul, Turkey

${ }^{8}$ Heller Institute of Medical Research and Medicine F, Sheba Medical Center, Tel Aviv, Israel

${ }^{9}$ UO Pediatria 2, Istituto G. Gaslini, Genova, Italy

${ }^{10}$ National Amyloidosis Centre UCL and Royal Free Hospital, London, UK

${ }^{11}$ Department of Radiation Oncology, Ankara Ataturk Chest Disease and Chest

Surgery Training and Research Hospital, Ankara, Turkey

${ }^{12}$ Department of Pediatric Pneumology and Immunology, Charite University Medicine

Berlin, Berlin, Germany

${ }^{13}$ Pediatric Rheumatology Unit, Hacettepe University Faculty of Medicine, Ankara,

Turkey

${ }^{14}$ National Human Genome Research Institute, National Institutes of Health,

Bethesda, Maryland, USA

${ }^{15}$ Instituto de Salud Musculoesquelética, Madrid, Spain

Contributors SO, ED, BE, AL, EB-C, GG, HO, IA, MG, PNH, SY, TK, YB, DK made substantial contributions to the conception or design of the work, or the acquisition, analysis or interpretation of data, drafting the work or revising it critically for important intellectual content.

Funding EULAR (grant no. CLI071).

Competing interests None declared.

Provenance and peer review Not commissioned; externally peer reviewed.

\section{REFERENCES}

1 Hentgen V, Grateau G, Kone-Paut I, et al. Evidence-based recommendations for the practical management of Familial Mediterranean Fever. Semin Arthritis Rheum 2013;43:387-91.

2 Kallinich T, Haffner D, Niehues T, et al. Colchicine use in children and adolescents with familial Mediterranean fever: literature review and consensus statement. Pediatrics 2007;119:e474-83.

3 van der Heijde D, Aletaha D, Carmona L, et al. 2014 Update of the EULAR standardised operating procedures for EULAR-endorsed recommendations. Ann Rheum Dis 2015;74:8-13.

4 Demirkaya E, Erer B, Ozen S, et al. Efficacy and safety of treatments in Familial Mediterranean Fever: a systematic review. Rheumatol Int 2015. doi:10.1007/ s00296-015-3408-9

5 Erer B, Demirkaya E, Ozen $\mathrm{S}$, et al. What is the best acute phase reactant for familial Mediterranean fever follow-up and its role in the prediction of complications? A systematic review. Rheumatol Int 2015. doi:10.1007/s00296-0153413-z

6 OCEBM Levels of Evidence Working Group. The Oxford 2011 Levels of Evidence. 2011. http://www.cebm.net/index.aspx?0=5653

7 Akar S, Yuksel F, Tunca M, et al. Familial Mediterranean fever: risk factors, causes of death, and prognosis in the colchicine era. Medicine 2012;91:131-6.

8 Duzova A, Bakkaloglu A, Besbas N, et al. Role of A-SAA in monitoring subclinical inflammation and in colchicine dosage in familial Mediterranean fever. Clin Exp Rheumatol 2003;21:509-14.

9 Livneh $A$, Zemer $D$, Langevitz $P$, et al. Colchicine treatment of AA amyloidosis of familial Mediterranean fever. An analysis of factors affecting outcome. Arthritis Rheum 1994:37:1804-11.

10 Livneh A, Zemer D, Siegal B, et al. Colchicine prevents kidney transplant amyloidosis in familial Mediterranean fever. Nephron 1992;60:418-22.

11 Zemer $D$, Pras $M$, Sohar $E$, et al. Colchicine in the prevention and treatment of the amyloidosis of familial Mediterranean fever. N Engl J Med 1986;314:1001-5.

12 Livneh A, Langevitz P. Diagnostic and treatment concerns in familial Mediterranean fever. Baillieres Best Pract Res Clin Rheumatol 2000;14:477-98.

13 Majeed $\mathrm{HA}$, Rawashdeh $\mathrm{M}$, el-Shanti $\mathrm{H}$, et al. Familial Mediterranean fever in children: the expanded clinical profile. QJM 1999;92:309-18.

14 Zemer D, Livneh A, Danon YL, et al. Long-term colchicine treatment in children with familial Mediterranean fever. Arthritis Rheum 1991;34:973-7.

15 Berkun $Y$, Wason S, Brik R, et al. Pharmacokinetics of colchicine in pediatric and adult patients with familial Mediterranean fever. Int J Immunopathol Pharmacol 2012:25:1121-30.

16 Padeh S, Shinar Y, Pras E, et al. Clinical and diagnostic value of genetic testing in 216 Israeli children with Familial Mediterranean fever. J Rheumatol 2003;30:185-90.
17 [No authors listed]. Ancient missense mutations in a new member of the RoRet gene family are likely to cause familial Mediterranean fever. The International FMF Consortium. Cell 1997;90:797-807.

18 Shohat M, Magal N, Shohat T, et al. Phenotype-genotype correlation in familial Mediterranean fever: evidence for an association between Met694Val and amyloidosis. Eur J Hum Genet 1999:7:287-92.

19 Cazeneuve C, Sarkisian T, Pêcheux C, et al. MEFV-Gene analysis in armenian patients with Familial Mediterranean fever: diagnostic value and unfavorable renal prognosis of the M694V homozygous genotype-genetic and therapeutic implications. Am J Hum Genet 1999;65:88-97.

20 Livneh $A$, Langevitz $P$, Shinar $Y$, et al. MEFV mutation analysis in patients suffering from amyloidosis of familial Mediterranean fever. Amyloid 1999:6:1-6.

21 Mimouni A, Magal N, Stoffman N, et al. Familial Mediterranean fever: effects of genotype and ethnicity on inflammatory attacks and amyloidosis. Pediatrics 2000;105:e70.

22 Medlej-Hashim M, Delague V, Chouery E, et al. Amyloidosis in familial Mediterranean fever patients: correlation with MEFV genotype and SAA1 and MICA polymorphisms effects. BMC Med Genet 2004;5:4.

23 Shinar $Y$, Livneh A, Langevitz P, et al. Genotype-phenotype assessment of common genotypes among patients with familial Mediterranean fever. J Rheumatol 2000;27:1703-7.

24 Ben-Chetrit E, Backenroth R. Amyloidosis induced, end stage renal disease in patients with familial Mediterranean fever is highly associated with point mutations in the MEFV gene. Ann Rheum Dis 2001;60:146-9.

25 Gershoni-Baruch R, Brik R, Zacks N, et al. The contribution of genotypes at the MEFV and SAA1 loci to amyloidosis and disease severity in patients with familial Mediterranean fever. Arthritis Rheum 2003;48:1149-55.

26 Ben-Chetrit E, Levy M. Colchicine: 1998 update. Semin Arthritis Rheum 1998;28:48-59.

27 Fradkin A, Yahav J, Zemer D, et al. Colchicine-induced lactose malabsorption in patients with familial Mediterranean fever. Isr J Med Sci 1995;31:616-20.

28 Lidar M, Livneh A. Familial Mediterranean fever: clinical, molecular and management advancements. Neth J Med 2007;65:318-24.

29 Levinger $U$, Monselise A. Reporting a desensitization protocol for colchicine treatment. Clin Exp Rheumatol 2001;19(Suppl 24):S79.

30 Cabili S, Shemer J, Revach $M$, et al. Allergic reactions and desensitization to colchicine in familial Mediterranean fever. Rheumatologie 1982;12:207-8.

31 Rozenbaum M, Boulman N, Feld J, et al. Intravenous colchicine treatment for six months: adjunctive therapy in familial Mediterranean fever (FMF) unresponsive to oral colchicine. Clin Exp Rheumatol 2009;27(Suppl 53):S105.

32 Lidar M, Kedem R, Langevitz $P$, et al. Intravenous colchicine for treatment of patients with familial Mediterranean fever unresponsive to oral colchicine. J Rheumatol 2003;30:2620-3.

33 Roldan R, Ruiz AM, Miranda MD, et al. Anakinra: new therapeutic approach in children with Familial Mediterranean Fever resistant to colchicine. Joint Bone Spine 2008:75:504-5.

34 Ben-Zvi I, Livneh A. Colchicine failure in familial Mediterranean fever and potential alternatives: embarking on the anakinra trial. Isr Med Assoc J 2014;16:271-3.

35 Hashkes PJ, Spalding SJ, Giannini EH, et al. Rilonacept for colchicine-resistant or -intolerant familial Mediterranean fever: a randomized trial. Ann Intern Med 2012;157:533-41.

36 Ozen S, Bilginer Y, Aktay Ayaz N, et al. Anti-interleukin 1 treatment for patients with familial Mediterranean fever resistant to colchicine. J Rheumatol 2011;38:516-18

37 Mitroulis I, Skendros P, Oikonomou A, et al. The efficacy of canakinumab in the treatment of a patient with familial Mediterranean fever and longstanding destructive arthritis. Ann Rheum Dis 2011;70:1347-8.

38 Seyahi E, Ozdogan H, Celik S, et al. Treatment options in colchicine resistant familial Mediterranean fever patients: thalidomide and etanercept as adjunctive agents. Clin Exp Rheumatol 2006;24(Suppl 42):S99-103.

39 Bilgen SA, Kilic L, Akdogan A, et al. Effects of anti-tumor necrosis factor agents for familial mediterranean fever patients with chronic arthritis and/or sacroiliitis who were resistant to colchicine treatment. J Clin Rheumatol 2011;17:358-62.

40 Twig G, Livneh A, Vivante A, et al. Mortality risk factors associated with familial Mediterranean fever among a cohort of 1.25 million adolescents. Ann Rheum Dis 2014;73:704-9.

41 Gillmore J, Lachmann H. Amyloidosis. In: Harber M, ed. Practical nephrology. London: Springer-Verlag, 2014:309-22.

42 Lachmann HJ. Secondary AA amyloidosis. In: Gertz MA, Vincent Rajkumar S, eds. Amyloidosis: diagnosis and treatment. Springer Science+Business Media, 2010:178-89.

43 Ansell D, Feest T, Byrne C, et al. UK renal registry report. Bristol: The Renal Association, 2003.

44 Keven K, Sengul S, Kutlay S, et al. Long-term outcome of renal transplantation in patients with familial Mediterranean fever amyloidosis: a single-center experience. Transplant Proc 2004;36:2632-4.

45 Erdem E, Karatas A, Kaya C, et al. Renal transplantation in patients with familial Mediterranean fever. Clin Rheumatol 2012;31:1183-6. 
46 Abedi AS, Nakhjavani JM, Etemadi J. Long-term outcome of renal transplantation in patients with familial Mediterranean fever amyloidosis: a single-center experience. Transplant Proc 2013;45:3502-4.

47 Yenokyan G, Armenian HK. Triggers for attacks in familial Mediterranean fever: application of the case-crossover design. Am J Epidemiol 2012;175:1054-61.

48 Karadag 0, Tufan A, Yazisiz V, et al. The factors considered as trigger for the attacks in patients with familial Mediterranean fever. Rheumatol Int 2013;33:893-7.

49 Fonnesu C, Cerquaglia C, Giovinale M, et al. Familial Mediterranean Fever: a review for clinical management. Joint Bone Spine 2009;76:227-33.

50 Ben-Chetrit E, Levy M. Reproductive system in familial Mediterranean fever: an overview. Ann Rheum Dis 2003;62:916-19.

51 Ben-Chetrit $\mathrm{E}$, Ben-Chetrit A. Familial Mediterranean fever and menstruation. BJOG 2001;108:403-7.

52 Piram $\mathrm{M}$, Koné-Paut I, Lachmann $\mathrm{HJ}$, et al. Validation of the auto-inflammatory diseases activity index (AIDAI) for hereditary recurrent fever syndromes. Ann Rheum Dis 2014;73:2168-73.

53 Konukbay D, Yildiz D, Acikel C, et al. Evaluation of bio-psycho-social aspects of patients with Juvenile autoinflammatory disease: a qualitative research. Ann Paediatr Rheum 2014;3:62-71.

54 Lachmann HJ, Sengül B, Yavuzşen TU, et al. Clinical and subclinical inflammation in patients with familial Mediterranean fever and in heterozygous carriers of MEFV mutations. Rheumatology (Oxford) 2006;45:746-50.

55 Ozdogan H, Ugurlu S, Hatemi G. Colchicine compliance and amyloidosis. Pediatric Rheumatol 2013;11(Suppl 1):A-15.

56 Tweezer-Zaks N, Doron-Libner A, Weiss P, et al. Familial Mediterranean fever and cryptogenic cirrhosis. Medicine (Baltimore) 2007;86:355-62.

57 Rimar D, Rosner I, Rozenbaum M, et al. Familial Mediterranean fever: an association with non-alcoholic fatty liver disease. Clin Rheumatol 2011;30:987-91.

58 Wilbur K, Makowsky M. Colchicine myotoxicity: case reports and literature review. Pharmacotherapy 2004;24:1784-92.

59 Ben-Chetrit $\mathrm{E}$, Backenroth $\mathrm{R}$, Levy M. Colchicine clearance by high-flux polysulfone dialyzers. Arthritis Rheum 1998;41:749-50.

60 Terkeltaub RA. Colchicine update: 2008. Semin Arthritis Rheum 2009;38: 411-19.

61 Ben-Chetrit E, Scherrmann JM, Zylber-Katz E, et al. Colchicine disposition in patients with familial Mediterranean fever with renal impairment. J Rheumatol 1994;21:710-13.

62 Terkeltaub RA, Furst DE, Digiacinto JL, et al. Novel evidence-based colchicine dose-reduction algorithm to predict and prevent colchicine toxicity in the presence of cytochrome P450 3A4/P-glycoprotein inhibitors. Arthritis Rheum 2011;63:2226-37.

63 Putterman C, Ben-Chetrit E, Caraco Y, et al. Colchicine intoxication: clinical pharmacology, risk factors, features, and management. Semin Arthritis Rheum 1991;21:143-55

64 Naidus RM, Rodvien R, Mielke CH Jr. Colchicine toxicity: a multisystem disease. Arch Intern Med 1977;137:394-6.

65 Baud FJ, Sabouraud A, Vicaut E, et al. Brief report: treatment of severe colchicine overdose with colchicine-specific Fab fragments. N Engl J Med 1995;332:642-5.

66 Ozturk MA, Kanbay M, Kasapoglu B, et al. Therapeutic approach to familial Mediterranean fever: a review update. Clin Exp Rheumatol 2011;29(Suppl 67):S77-86.
67 Amital H, Ben-Chetrit E. Therapeutic approaches to familial Mediterranean fever. What do we know and where are we going to? Clin Exp Rheumatol 2004;22(Suppl 34):S4-7.

68 Meinzer U, Quartier P, Alexandra JF, et al. Interleukin-1 targeting drugs in familial Mediterranean fever: a case series and a review of the literature. Semin Arthritis Rheum 2011:41:265-71.

69 Ben-Chetrit E, Scherrmann JM, Levy M. Colchicine in breast milk of patients with familial Mediterranean fever. Arthritis Rheum 1996;39:1213-17.

70 Taylor EW. The mechanism of colchicine inhibition of mitosis. I. Kinetics of inhibition and the binding of H3-colchicine. J Cell Biol 1965;25(Suppl):145-60.

71 Barsoum $\mathrm{H}$. The effect of colchicine on the spermatogenesis of rabbits. J Pharmacol Exp Ther 1955;115:319-22.

72 Merlin HE. Azoospermia caused by colchicine-a case report. Fertil Steril 1972;23:180-1

73 Cohen MM, Levy M, Eliakim M. A cytogenic evaluation of long-term colchicine therapy in the treatment of Familial Mediterranean fever (FMF). Am J Med SCi 1977;274:147-52.

74 Bremner WJ, Paulsen CA. Colchicine and testicular function in man. N Engl J Med 1976:294:1384-5.

75 Haimov-Kochman R, Prus D, Ben-Chetrit E. Azoospermia due to testicular amyloidosis in a patient with familial Mediterranean fever. Hum Reprod 2001;16:1218-20.

76 Ben-Chetrit $E$, Berkun $Y$, Ben-Chetrit $E$, et al. The outcome of pregnancy in the wives of men with familial mediterranean fever treated with colchicine. Semin Arthritis Rheum 2004;34:549-52.

77 Langevitz $P$, Livneh A, Zemer D, et al. Seronegative spondyloarthropathy in familial Mediterranean fever. Semin Arthritis Rheum 1997;27:67-72.

78 Sakallioglu O, Duzova A, Ozen S. Etanercept in the treatment of arthritis in a patient with familial Mediterranean fever. Clin Exp Rheumatol 2006;24:435-7.

79 Kaplan E, Mukamel M, Barash J, et al. Protracted febrile myalgia in children and young adults with familial Mediterranean fever: analysis of 15 patients and suggested criteria for working diagnosis. Clin Exp Rheumatol 2007;25(Suppl 45): S114-17.

80 Sidi G, Shinar Y, Livneh A, et al. Protracted febrile myalgia of familial Mediterranean fever. Mutation analysis and clinical correlations. Scand I Rheumatol 2000;29:174-6

81 Majeed HA, Al-Qudah AK, Qubain $\mathrm{H}$, et al. The clinical patterns of myalgia in children with familial Mediterranean fever. Semin Arthritis Rheum 2000;30:138-43.

82 Langevitz $\mathrm{P}$, Zemer D, Livneh A, et al. Protracted febrile myalgia in patients with familial Mediterranean fever. J Rheumatol 1994;21:1708-9.

83 Mercan $R$, Turan A, Bitik B, et al. Rapid resolution of protracted febrile myalgia syndrome with anakinra: report of two cases. Mod Rheumatol 2014. doi:10.3109/ 14397595.2014.882221

84 Ben-Zvi I, Krichely-Vachdi T, Feld 0, et al. Colchicine-free remission in familial Mediterranean fever: featuring a unique subset of the disease-a case control study. Orphanet J Rare Dis 2014;9:3

85 Giancane G, Ter Haar NM, Wulffraat N, et al. Evidence-based recommendations for genetic diagnosis of familial Mediterranean fever. Ann Rheum Dis 2015;74:635-41.

86 Shinar Y, Obici L, Aksentijevich I, et al. Guidelines for the genetic diagnosis of hereditary recurrent fevers. Ann Rheum Dis 2012;71:1599-605. 